\title{
Control visual para la formación de robots móviles tipo uniciclo bajo el esquema líder-seguidor
}

\author{
Visual Control for Unicycle-Like Mobile Robots Formation \\ Under the Leader-Follower Scheme
}

\author{
Bugarin-Carlos Eusebio \\ División de Estudios de Posgrado e Investigación \\ Instituto Tecnológico de Ensenada, B.C. \\ Correo:eusebio@hotmail.com
}

\author{
Aguilar-Bustos Ana Yaveni \\ División de Estudios de Posgrado e Investigación \\ Instituto Tecnológico de Ensenada, B.C. \\ Correo:yaveni@hotmail.com
}

Información del artículo: recibido: abril de 2013, reevaluado: mayo de 2013, aceptado: septiembre de 2013

\section{Resumen}

El presente trabajo describe una propuesta de control visual para la formación de robots móviles tipo uniciclo bajo el esquema líder-seguidor. Se considera una sola cámara fija observando el espacio de trabajo de los robots que, en términos de la información procesada, puede ser compartida tanto por el robot líder como por el robot seguidor. Lo anterior permitiría que la realización de esta propuesta pueda llevarse a cabo por estrategias de control centralizadas o descentralizadas. Para efectos de simplificar el análisis, también se considera que el plano de imagen es paralelo al plano de movimiento de los robots. El objetivo de formación se establece directamente en coordenadas de imagen y el controlador visual propuesto no depende explícitamente de los parámetros (extrínsecos o intrínsecos) del sistema de visión; lo que en conjunto corresponde a la contribución principal de este artículo. Por último, también como parte importante de este trabajo, para validar la teoría propuesta se detallan experimentos satisfactorios utilizando un sistema de visión de tiempo real y alta velocidad.

\footnotetext{
Abstract

This paper describes a visual control proposal for the formation of unicycle-like mobile robots under the leader-follower scheme. It is considered a single fixed camera observing the robots workspace that, in terms of the processed information, can be shared by both the leader robot and the follower robot. This would enable the implementation of this proposal to be performed by centralized or decentralized control strategies. For the purpose of simplifying the analysis, it is also considered that the image plane is parallel to the robots motion plane. The formation objective is established directly in image space and the proposed visual controller does not depend explicitly on the vision system parameters (extrinsic or intrinsic); which together represents the main contribution of this paper. Finally, also as an important part of this work, to validate the proposed theory satisfactory experiments using a real-time and high-speed vision system are detailed.
}

\section{Descriptores:}

- formación de robots

- control visual

- robots uniciclo

- control de robots

- validación experimental

\section{Keywords:}

- robot formation

- visual control

- unicycle robots

- robot control

- experimental validation 


\section{Introducción}

El problema de formación de robots consiste en establecer el movimiento de un grupo de robots para que, de una manera coordinada o colaborativa, lleven a cabo una tarea específica (Das et al., 2002). Recientemente este problema ha atraído significativamente la atención de la comunidad científica, ya sea por el reto de sus espacios de trabajo no estructurados o por las aplicaciones complejas que se pueden resolver. Existen ciertas tareas que son difíciles de lograr por un solo robot o que son más eficientes si se llevan a cabo mediante la coordinación de un grupo de robots. Entre los ejemplos de esas tareas tenemos: vigilancia, búsqueda de objetos, exploración, rescate y transportación de objetos; las cuales pueden realizarse en ambientes diversos utilizando robots móviles terrestres, aéreos, espaciales, marinos o submarinos.

En la literatura se mencionan diversos métodos para resolver el problema de formación de robots; destacándose los "basados en comportamiento" (Balch y Arkin, 1998; Lawton et al., 2003; Antonelli et al., 2006) en donde, precisamente, diferentes comportamientos, como mantener una formación o seguir un objetivo, se imponen a cada robot (en este método el control de formación exacto es difícil de garantizar); los de "estructura virtual" (Tan y Lewis, 1997; Belta y Kumar, 2002) que consideran al grupo de robots como una sola estructura rígida virtual (aquí es necesaria una comunicación interrobots muy amplia); y los de "líder-seguidor" (Desai et al., 2001; Monteiro et al., 2004; Shao et al., 2005; Consolini et al., 2006) en donde uno o varios robots se designan como los líderes de la formación y el resto como los seguidores, a los cuales se les especifica la postura (posición y orientación) deseada relativa al o los líderes. Este último método es de particular interés debido a su simplicidad y modularidad (Consolini et al., 2006).

Ahora bien, debido a que el espacio de trabajo en este problema de formación de robots es difícil de estructurar se hace necesaria la incorporación de sensores exteroceptivos (además de los propioceptivos o internos de cada robot) que midan de alguna manera tanto el entorno de interacción como las posturas de los demás robots en la formación. Esta situación se ha resuelto utilizando sistemas de posicionamiento global (GPSs), sistemas RADAR (mediante ondas de radio electromagnéticas), sistemas LIDAR (a través de detección láser) o mediante sistemas de visión (Benhimane et al., 2005; Mehta et al., 2006). Sin embargo, dados los recientes avances tecnológicos, los sistemas de visión están siendo cada vez más utilizados.
El uso de un sistema de visión para determinar el movimiento de un sistema robótico se denomina control visual o servo-visual (visual servoing) desde la propuesta original de Hill y Park (1979). Básicamente, existen dos alternativas para el control visual: la "basada en posición", en donde la sucesión de imágenes se emplea para reconstruir el espacio tridimensional de trabajo; y la "basada en imagen", en la cual el objetivo de control se da directamente en el espacio de imagen; de manera que en esta última alternativa se incrementa la posibilidad de no depender explícitamente de los parámetros extrínsecos (los que tienen que ver con la postura de la o las cámaras) o intrínsecos (los que tiene que ver con la estructura interna de la o las cámaras) del sistema de visión (Hutchinson et al., 1996).

En el presente trabajo se describe la propuesta de un controlador visual basado en imagen para la formación de dos robots móviles bajo el esquema líderseguidor. Los robots considerados corresponden a los robots móviles terrestres tipo uniciclo, los cuales se caracterizan por tener dos ruedas convencionales con actuadores independientes y una tercera rueda sin actuador para mantener su equilibrio horizontal. Esto los convierte en sistemas no-holonómicos que presentan ciertas propiedades interesantes; por ejemplo, el sistema linealizado es no-controlable, por lo que los métodos lineales de análisis y diseño no pueden aplicarse; tampoco existe una ley de control continua que incluya solo retroalimentación de estados capaz de estabilizar el sistema a un estado de equilibrio (Brockett, 1983). Trabajos relacionados con el objetivo de control de postura de un robot móvil mediante el control visual los podemos ver en Hashimoto y Noritsugu (1997), Conticelli et al. (1999), Mariottini et al. (2004), Fang et al. (2005) y López-Nicolás et al. (2006).

En particular, sobre el control visual con el objetivo de formación de robots móviles bajo el esquema líderseguidor, se pueden mencionar las propuestas de Renaud et al. (2004), Benhimane et al. (2005), Soria et al. (2006) y Min et al. (2009); las cuales emplean la alternativa basada en posición con cámara montada y con la necesidad del conocimiento total o parcial de los parámetros del sistema de visión. En Das et al. (2002) y Roberti et al. (2011) se proponen controladores con las mismas condiciones anteriores, pero utilizando sistemas de visión catadióptricos, básicamente para ampliar el campo de visión. En Dani et al. (2009) se describe un trabajo también basado en posición y con cámara montada, pero eliminando totalmente la necesidad del conocimiento de los parámetros del sistema de visión. 


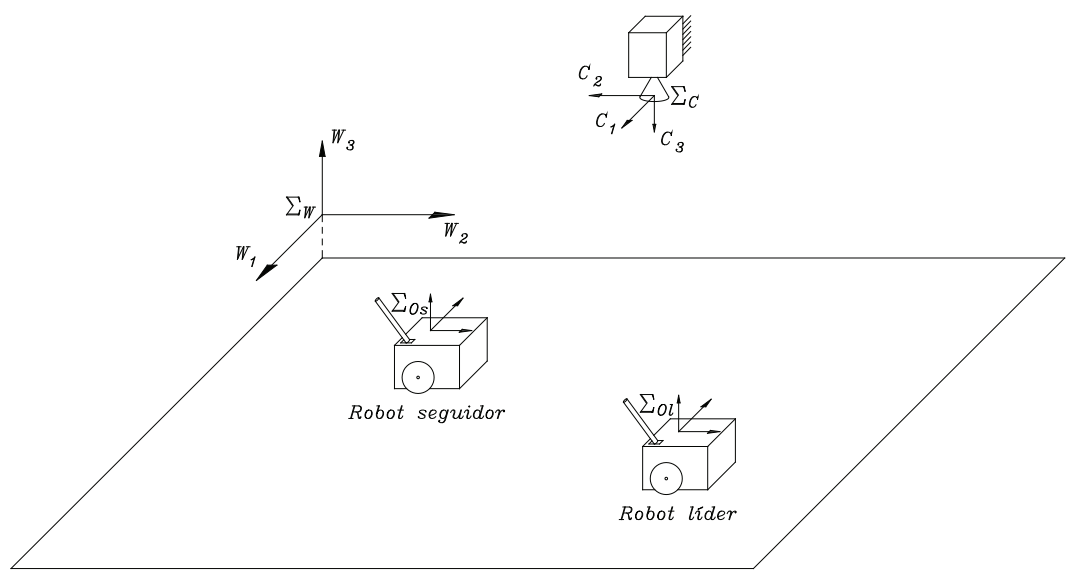

Figura 1. Disposición del sistema robótico

A diferencia de los trabajos recién citados, la disposición del sistema robótico en este documento considera una cámara fija observando todo el espacio de trabajo de los robots (figura 1) con la alternativa de control visual basada en imagen. Lo anterior va encaminado a la eliminación de los problemas de oclusión y la facilidad de establecer las variables de formación deseadas, sin necesidad de realizar medición tridimensional (en coordenadas de mundo $\Sigma_{W}$ ) alguna. Dependiendo de la aplicación, dicha cámara puede estar instalada (o fija) en un globo aerostático, en un edificio o en alguna parte alta del entorno. Ahora bien, uno de los propósitos es que el sistema de visión sea compartido tanto por el robot líder como por el robot seguidor, de tal suerte que la implementación de la presente propuesta se pueda lograr por esquemas de control, tanto centralizados como descentralizados. Para efectos de simplificar el análisis, también se considera que el plano de imagen es paralelo al plano horizontal de movimiento de los robots. De esta manera, explícitamente el objetivo de formación se establece de manera directa en coordenadas de imagen y se logra que el controlador visual propuesto no dependa explícitamente de los parámetros (extrínsecos o intrínsecos) del sistema de visión; lo que en conjunto corresponde a la contribución principal de este artículo. Por último, también como parte importante de este trabajo, para validar la teoría propuesta se detallan experimentos satisfactorios utilizando un sistema de visión de tiempo real y alta velocidad.

\section{Formulación}

En este apartado se describe la formulación de la propuesta del controlador visual para la formación de robots uniciclos en el esquema líder-seguidor.
Modelo de imagen

Considere la vista de planta del sistema robótico mostrada en la figura $2^{1}$. Observe que se han colocado diversos marcos coordenados (compare las figuras 1 y 2 ): $\Sigma_{C}$ el marco de la cámara con origen en el centro de la lente, $\Sigma_{W}$ el marco de mundo fijo en algún lugar del espacio de trabajo a una altura conveniente, $\Sigma_{O l}$ el marco del líder y $\Sigma_{O S}$ el marco del seguidor (estos dos últimos marcos con origen en el centro del eje que une sus dos ruedas a una altura convenientemente nula). Note que los ejes " 3 " de cada marco son paralelos (debido a la postura de la cámara utilizada), por lo que los planos $W_{1}-W_{2}, C_{1}-C_{2}, O_{l 1}-O_{l 2}$ y $O_{s 1}-$ $O_{s 2}$ también son paralelos (tanto $O_{l 1}$ como $O_{s 1}$ apuntan en la dirección de movimiento de cada uniciclo respectivo); sin embargo, puede existir rotación entre cada uno de estos planos.

Con base en la estrategia de control visual o servovisual utilizada, considere como objetos de interés (Hutchinson et al., 1996) dos discos (de radios arbitrarios, pero con la condición de que sus centroides puedan ser procesados por el sistema de visión) localizados sobre los robots móviles (figura 2): el disco $a_{1}$ del líder con centro $x_{\text {Ola }}=\left[\begin{array}{ll}l_{O} & 0\end{array}\right]^{T}[\mathrm{~m}]$, el disco $b_{l}$ del líder con centro en $x_{\mathrm{Olb}}=\left[\begin{array}{ll}0 & 0\end{array}\right]^{T}[\mathrm{~m}]$ (los subíndices $\mathrm{Ol}$ denotan que son vectores de posición respecto al marco $\Sigma_{O I}$ ) y los discos $a_{\mathrm{s}}$ y $b_{\mathrm{s}}$ del seguidor puestos de manera similar, es decir, con $x_{\text {Osa }}=\left[\begin{array}{ll}l_{O} & 0\end{array}\right]^{T}[\mathrm{~m}]$ y $x_{\text {Osb }}=\left[\begin{array}{ll}0 & 0\end{array}\right]^{T}[\mathrm{~m}]$ (los subíndices $O s$ denotan que son vectores de posición respecto al marco $\left.\Sigma_{O_{s}}\right)$; donde la distancia $l_{O}>0$ es una constante.

Ahora bien, el modelo de imagen considerado para el mapeo hacia el plano de imagen $y_{1}-y_{2}$ es el que corresponde al obtenido mediante trasformaciones y proyección de perspectiva con lente delgada, dado por (Kelly y Reyes, 2000):

$y=\left[\begin{array}{l}y_{1} \\ y_{2}\end{array}\right]=\frac{\alpha_{C} \lambda_{C}}{x_{C 3}-\lambda_{C}}\left[\begin{array}{l}x_{C 1} \\ x_{C 2}\end{array}\right]+\left[\begin{array}{l}u_{C} \\ v_{C}\end{array}\right]$

donde $x_{\mathrm{C}}=\left[\begin{array}{lll}x_{\mathrm{C} 1} & x_{\mathrm{C} 2} & x_{\mathrm{C} 3}\end{array}\right]^{T}$ es el punto correspondiente con coordenadas tridimensionales respecto al marco $\Sigma_{C}$ de la cámara, $\alpha_{C}$ es el factor de conversión de metros a píxeles (considerándose el mismo factor de conversión tanto para $y_{1}$ como para $\left.y_{2}\right), \lambda_{c}$ es la distancia focal de la

1 Cada variable de esta figura se describirá en su momento. 
lente y $\left[\begin{array}{ll}u_{C} & v_{C}\end{array}\right]^{T}$ es el vector que incluye el centro de la imagen y las posibles desalineaciones entre el eje óptico de la cámara y su arreglo de sensores fotosensibles. Observe que $y_{1}$ siempre es paralelo y apunta en la misma dirección que $C_{1}$ (lo mismo sucede con $y_{2}$ y $C_{2}$; consulte la figura 2).

La transformación de coordenadas entre el marco del mundo y el marco de la cámara se puede realizar mediante

$x_{C}=R_{W}^{C^{T}}(\phi)\left[x_{W}-\mathrm{O}_{W}^{C}\right]$

donde $x_{W}=\left[\begin{array}{lll}x_{W 1} & x_{W 2} & x_{W 3}\end{array}\right]^{T}$ es el punto tridimensional correspondiente respecto a $\Sigma_{W}, \mathrm{O}_{W}^{C}$ es el vector de posición del origen de $\Sigma_{C}$ respecto a $\Sigma_{W} W_{X} \mathrm{y}$

$R_{W}^{\mathrm{C}}(\phi)=\left[\begin{array}{ccc}\cos (\phi) & \operatorname{sen}(\phi) & 0 \\ \operatorname{sen}(\phi) & -\cos (\phi) & 0 \\ 0 & 0 & -1\end{array}\right]$

es la matriz de rotación de $\Sigma_{C}$ respecto a $\Sigma_{W}(\pi$ [rad] alrededor de $W_{1}$ y $\phi$ [rad] alrededor de $W_{3}$, por la probable rotación "desconocida" pero constante entre los planos $W_{1}-W_{2}$ y $\left.C_{1}-C_{2}\right)$.

Modelo de los uniciclos

El robot líder y el robot seguidor considerados corresponden a los que se componen de dos ruedas convencionales con actuadores independientes y una tercer rueda sin actuador para mantener su equilibrio horizontal y que poseen el siguiente modelo cinemático (Canudas de Wit et al., 1996; Dixon et al., 2001):

$\frac{d}{d t}\left[\begin{array}{l}x_{W i 1} \\ x_{W i 2} \\ \theta_{i}\end{array}\right]=\left[\begin{array}{cc}\cos \left(\theta_{i}\right) & 0 \\ \operatorname{sen}\left(\theta_{i}\right) & 0 \\ 0 & 1\end{array}\right]\left[\begin{array}{l}u_{i 1} \\ u_{i 2}\end{array}\right], i=\{l, s\}$

donde $l$ y $s$ son subíndices para denotar correspondencia con el robot líder o con el robot seguidor, respectivamente. El vector $x_{W i}=\left[\begin{array}{lll}x_{W i 1} & x_{W i 2} & 0\end{array}\right]^{T}$ denota la posición del robot uniciclo $i$ (el punto medio del eje que une las dos ruedas a una altura convenientemente nula, ver figuras 1 y $2, \theta_{i}$ la orientación del uniciclo $i$, ambos respecto a $\Sigma_{W}$. Las componentes del vector de entrada $u_{i}=\left[\begin{array}{ll}u_{i 1} & u_{i 2}\end{array}\right]^{T}$ son la magnitud de la velocidad lineal (a lo largo de $O_{i 1}$ ) y angular (alrededor de $O_{i 3}$ ) del robot $i$, respectivamente.

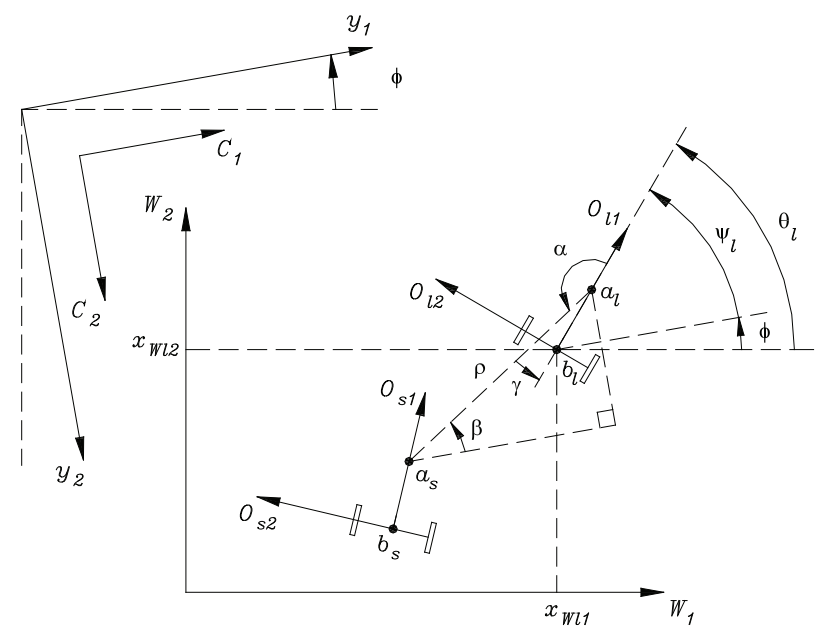

Figura 2. Vista de planta del sistema robótico

\section{Variables de formación}

Las variables de formación serán las referencias mediante las que se establecerá formalmente el objetivo de formación de los robots uniciclo, estas variables de formación estarán dadas directamente en coordenadas de imagen. Para esto, considere como variables de formación a $\rho$ y $\alpha$, donde (figura 2 )

$\rho=\left\|y_{a l}-y_{a s}\right\|$

es la distancia en píxeles entre las proyecciones en el plano de imagen de los centroides de los discos $a$ de los robots líder y seguidor, y $\alpha$ la orientación de la formación. Obsérvese en la figura 2 que

$$
\begin{aligned}
\alpha+\gamma & =\pi \\
\alpha & =\pi-\gamma \\
\alpha & =\pi-\left[\pi / 2-\beta-\left[\pi / 2-\psi_{l}\right]\right] \\
\alpha & =\pi-\psi_{l}+\beta
\end{aligned}
$$

donde $\gamma$ es un ángulo auxiliar, $\psi_{l}=\theta_{l}-\phi$ es la orientación del uniciclo líder en el plano de imagen y

$\beta=\tan ^{-1}\left(\frac{-\left[y_{a l 2}-y_{a s 2}\right]}{y_{a l 1}-y_{a s 1}}\right)$

es el ángulo formado entre el eje $y_{1}$ y la línea formada por $y_{a l}$ y $y_{a s}$ (para la cálculo de las variables $\alpha, \beta$ y $\psi_{l}$ se recomienda utilizar la función atan2, ver por ejemplo Spong et al. (2006) y tener especial cuidado para que $\alpha, \beta, \Psi_{l} \in \mathfrak{R}$ de manera que no se les limite a $\pm \pi$ [rad]). 


\section{Controlador}

En esta sección se detallan el modelo a controlar y el diseño del controlador visual para la formación de uniciclos además de su prueba de estabilidad.

\section{Modelo a controlar}

El modelo a controlar corresponderá a la dinámica desarrollada por dos señales de error relacionadas con las variables de formación (4) y (5). En esta búsqueda, definimos el siguiente vector de error

$e=\left[\begin{array}{l}e_{\rho} \\ e_{\alpha}\end{array}\right]=\left[\begin{array}{c}\rho_{d}-\rho \\ \alpha_{d}-\alpha\end{array}\right]$

donde $\rho_{d}$ y $\alpha_{d}$ son la distancia (en píxeles) y la orientación deseadas (y constantes) de la formación, respectivamente. De manera que la derivada respecto al tiempo de (7) resulta

$\dot{e}=\left[\begin{array}{c}-\dot{\rho} \\ -\dot{\alpha}\end{array}\right]$,

por lo que es necesario derivar respecto al tiempo (4) y (5). Antes de encontrar las derivadas temporales, primero observe que un punto $x_{\mathrm{O} i}$ respecto al marco $\Sigma_{\mathrm{O} i}$ (con $i=\{l, s\}$ ) puede transformarse hacia el marco $\Sigma_{W}$ mediante

$x_{W}=R_{W}^{O_{i}}\left(\theta_{i}\right) x_{O i}+O_{W}^{O_{i}}$

donde el vector $O_{W}^{O_{i}}$ denota el vector de posición del origen de $\Sigma_{O i}$ respecto a $\Sigma_{W} \mathrm{y}$

$R_{W}^{O_{i}}\left(\theta_{i}\right)=\left[\begin{array}{ccc}\cos \left(\theta_{i}\right) & -\operatorname{sen}\left(\theta_{i}\right) & 0 \\ \operatorname{sen}\left(\theta_{i}\right) & \cos \left(\theta_{i}\right) & 0 \\ 0 & 0 & 1\end{array}\right]$

representa la matriz de rotación (en función de $\theta_{i}$ ) del marco $\Sigma_{O i}$ respecto a $\Sigma_{W}$. De esta manera, utilizando (2), (3) y (9) se encuentra que la derivada temporal de (1) es (ecuación 10) donde $\psi_{i}=\theta_{i}-\phi$ es la orientación del robot $i$ en el plano de imagen.

Ahora, siguiendo la metodología propuesta en $\mathrm{Bu}-$ garin y Kelly (2008), para simplificar (10) definimos $\bar{y}_{i}=y_{a i}-y_{b i}$, de modo que utilizando (1), (2) y (9) se puede llegar a lo siguiente

$\bar{y}_{i}=\frac{\alpha_{c} \lambda_{c}}{x_{\text {Cai3 }}-\lambda_{c}}\left[\begin{array}{r}l_{O} \cos \left(\psi_{i}\right) \\ -l_{O} \operatorname{sen}\left(\psi_{i}\right)\end{array}\right], i=\{1, \mathrm{~s}\}$

puesto que $x_{\text {Cai3 }}=x_{\text {Cbi3 }}$. En consecuencia, despejando $\operatorname{sen}\left(\psi_{i}\right)$ y $\cos \left(\psi_{i}\right)$ de (11) se puede simplificar (10) para la variación respecto al tiempo de $y_{a i}$ llegando al siguiente resultado

$\dot{y}_{a i}=\left[\begin{array}{cc}\frac{\bar{y}_{i 1}}{l_{O}} & \bar{y}_{i 2} \\ \frac{\bar{y}_{i 2}}{l_{O}} & -\bar{y}_{i 1}\end{array}\right]\left[\begin{array}{l}u_{i 1} \\ u_{i 2}\end{array}\right], \quad i=\{1, \mathrm{~s}\}$,

es decir, con las condiciones especificadas, la variación respecto al tiempo de $y_{a i}$ no depende de los parámetros (intrínsecos o extrínsecos) de la cámara. Finalmente, después de derivar respecto al tiempo (4) y (5) utilizando (12), el modelo a controlar (8) se simplifica a

$$
\dot{e}=A\left(y ; l_{O}\right)\left[\begin{array}{l}
u_{l 1} \\
u_{l 2}
\end{array}\right]+B\left(y ; l_{O}\right)\left[\begin{array}{l}
u_{s 1} \\
u_{s 2}
\end{array}\right]
$$

donde

$$
\begin{aligned}
& A\left(y ; l_{O}\right)=\left[\begin{array}{ll}
\frac{\bar{y}_{l s 2} \bar{y}_{l 2}+\bar{y}_{l 1} \bar{y}_{l 1}}{-l_{O} \rho} & \frac{\bar{y}_{l s} \bar{y}_{l 1}-\bar{y}_{l s 1} \bar{y}_{l 2}}{\rho} \\
\frac{\bar{y}_{l s} \bar{y}_{l 2}-\bar{y}_{l s 2} \bar{y}_{l 1}}{l_{O} \rho^{2}} & \frac{\bar{y}_{l s 1} \bar{y}_{l 1}+\bar{y}_{l s 2} \bar{y}_{l 2}}{-\rho^{2}}
\end{array}\right], \\
& B\left(y ; l_{O}\right)=\left[\begin{array}{ll}
\frac{\bar{y}_{l s 2} \bar{y}_{s 2}+\bar{y}_{l s 1} \bar{y}_{s 1}}{-l_{O} \rho} & \frac{\bar{y}_{l s 2} \bar{y}_{s 1}-\bar{y}_{l s 1} \bar{y}_{s 2}}{\rho} \\
\frac{\bar{y}_{l 11} \bar{y}_{s 2}-\bar{y}_{l s 2} \bar{y}_{s 1}}{-l_{O} \rho^{2}} & \frac{\bar{y}_{l 1} \bar{y}_{s 1}+\bar{y}_{l s 2} \bar{y}_{s 2}}{\rho^{2}}
\end{array}\right],
\end{aligned}
$$

$\dot{y}_{i}=\frac{\alpha_{c} \lambda c}{x_{\mathrm{Ci3}}-\lambda_{c}}\left[\begin{array}{rr}\cos \left(\psi_{i}\right) & -\operatorname{sen}\left(\psi_{i}\right) x_{\mathrm{O} 11}-\cos \left(\psi_{i}\right) x_{\mathrm{Oi2}} \\ -\operatorname{sen}\left(\psi_{i}\right) & -\cos \left(\psi_{i}\right) x_{\mathrm{O} i 1}+\operatorname{sen}\left(\psi_{i}\right) x_{\mathrm{Oi2}}\end{array}\right]\left[\begin{array}{c}u_{i 1} \\ u_{i 2}\end{array}\right], i=\{l, s\}$ 
con $\bar{y}_{l s}=y_{a l}-y_{a s}$. Cabe destacar que el determinante de $\mathrm{B}\left(y ; l_{0}\right)$ es igual a $\frac{\left\|y_{s}\right\|}{l_{0} \rho}$, en donde tanto $\left\|\bar{y}_{S}\right\|$ (la distancia en píxeles entre los centroides de los discos $a_{s}$ y $b_{s}$ del robot seguidor) como $l_{O}$ son constantes, por lo que si $\rho \neq 0$ entonces existe la inversa de $B\left(y ; l_{O}\right)$.

Objetivo del control de formación y diseño del controlador

El objetivo del control de formación queda formalmente establecido en relación a (7) mediante

$\lim _{t \rightarrow \infty} e(t)=0$,

es decir, se desea que las variables de formación, especificadas directamente en coordenadas de imagen, lleguen asintóticamente a un valor constante deseado.

Por hipótesis supóngase que existe la inversa de $B\left(y ; l_{0}\right)$, entonces para resolver el objetivo de control (14) recién descrito, el presente trabajo propone el siguiente controlador visual para el robot seguidor

$u_{s}=-B\left(y ; l_{0}\right)^{-1}\left[K e+A\left(y ; l_{0}\right) u_{l}\right]$

donde $K=K^{T}>0$ es una matriz de ganancia. Note que este controlador no depende de los parámetros (intrínsecos o extrínsecos) del sistema de visión y que puede ponerse en marcha completamente solo con mediciones directas en el plano de imagen. Asimismo, debe comentarse que se necesita la transmisión de las velocidades del líder al seguidor (trabajos recientes, como el de Dani et al., (2009), van en la dirección de estimar dichas velocidades para eliminar la necesidad de su transmisión; en el presente artículo esto se considera como un trabajo futuro) y del parámetro $l_{O}$ (el cual es un parámetro determinado por el propio usuario).

Para la prueba de estabilidad, se supone válida la existencia de la inversa de $B\left(y ; l_{0}\right)$, luego se sustituye la ley de control (15) en el modelo (13) para encontrar la ecuación de lazo cerrado del sistema robótico, la cual queda expresada por

$\dot{e}=-K e$.

De esta manera, puede observarse que la ecuación del sistema en lazo cerrado corresponde a un sistema lineal e invariante en el tiempo (o autónomo) y debido a que por diseño $\mathrm{K}=\mathrm{K}^{\mathrm{T}}>0$ el origen es su único punto de equilibrio con la propiedad de ser exponencialmente estable de manera global (vea por ejemplo Khalil (2001)); de esta manera se demuestra que el objetivo de control (14) es satisfecho. Con esto también se demuestra que al tender los errores exponencialmente a cero, $\rho$ tiende exponencialmente a $\rho_{\mathrm{d}}$, de manera que si la condición inicial para $\rho(0) \neq 0$ y $\rho_{\mathrm{d}}>0$ entonces $\rho(t) \neq 0$ $\forall t>0$; lo que implica que la suposición inicial de que la inversa de $\mathrm{B}\left(\mathrm{y} ; \mathrm{l}_{\mathrm{O}}\right)$ existe es válida.

Cabe mencionar que en todo el análisis realizado se ha considerado el sistema robótico como un sistema continuo y determinista, por lo que la velocidad del robot líder $u_{l}$ especificada en (15) debe ser continuamente diferenciable. Por otro lado y para cuestiones prácticas, puede considerarse que el valor de $\rho$ siempre tendrá una magnitud aceptable que no afectaría el cálculo de las matrices $A\left(y ; l_{0}\right)$ y $B\left(y ; l_{0}\right)$, puesto que de inicio debe existir una distancia mínima suficiente entre los robots para que estos físicamente no estén uno sobre el otro.

\section{Validación experimental}

Para validar la teoría propuesta, en este apartado se exponen experimentos desarrollados con dos robots uniciclo modelo YSR-A de la empresa Yujin. Dichos robots reciben las consignas de velocidad a través de un transmisor RF que se conecta a una computadora personal vía puerto serie. A esta misma computadora se le conecta (vía Camera Link), mediante una tarjeta videoprocesadora Leonardo de la empresa Arvoo, una cámara de video digital de alta velocidad modelo UF$1000 C L$ de la empresa UNIQ. Sobre el sistema operativo RTLinux se desarrolla un sistema de control de tiempo real para el procesamiento de las imágenes y la ley de control, obteniéndose un periodo de muestreo estricto de 0.005 [s]. Cabe mencionar que el procesamiento de las imágenes se lleva a cabo mediante segmentación binaria y seguimiento de la característica de imagen (el centroide de los discos).

A continuación se detallan dos escenarios que corresponden a las siguientes variables de formación deseadas:

$\rho_{d}=70$ [píxeles] y $\alpha_{d}=90^{\circ}$,

con la ganancia en el controlador

$K=\left[\begin{array}{ll}4 & 0 \\ 0 & 4\end{array}\right]$

y con la distancia entre los centros de los discos objetivo $l_{O}=0.0375[\mathrm{~m}]$. Estos 4 valores son los únicos necesarios para la experimentación.

Ahora bien, solamente para efectos de comparación, se realizaron simulaciones correspondientes con los siguientes parámetros del sistema de visión: $\alpha_{C}=72000$ 
[píxeles $/ \mathrm{m}], \lambda_{C}=0.0075[\mathrm{~m}], u_{C}=160$ [píxeles], $v_{C}=120$ [píxeles], $O_{W}^{C}=\left[\begin{array}{lll}0 & 0 & 1.5\end{array}\right]^{\mathrm{T}}[\mathrm{m}]$ y $\phi=0[\mathrm{rad}]$.

\section{Escenario 1}

Este escenario 1 se experimentó con velocidades del líder nulas, es decir, con $u_{l}=0$ y con las siguientes condiciones iniciales

$x_{W I}(0)=\left[0.0758 \mathrm{~m}-0.0086 \mathrm{~m} 43.31^{\circ}\right]^{T}$,

$x_{W_{s}}(0)=\left[\begin{array}{lll}0.0837 \mathrm{~m} & 0.2750 \mathrm{~m} & 177.92^{\circ}\end{array}\right]^{T}$.

Estas condiciones iniciales corresponden a (en píxeles) $y_{a l}(0)=\left[\begin{array}{ll}197.3 & 113.8\end{array}\right]^{T}, y_{b l}(0)=\left[\begin{array}{ll}187.4 & 123.1\end{array}\right]^{T}, y_{a s}(0)=$ $\left[\begin{array}{ll}176.720 .0\end{array}\right]^{T}, y_{b s}(0)=\left[\begin{array}{ll}190.3 & 20.5\end{array}\right]^{T}, \rho(0)=96.0$ y $\alpha(0)=$ $59.05^{\circ}$.

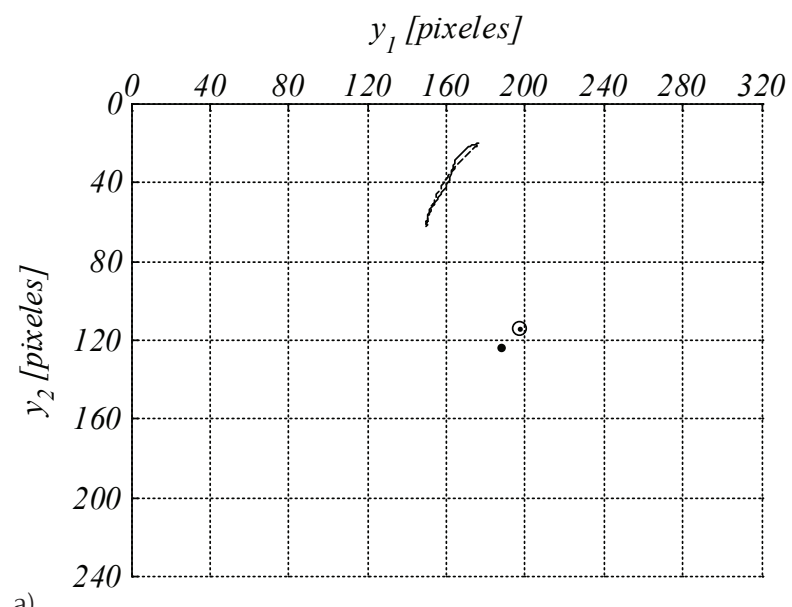

a)

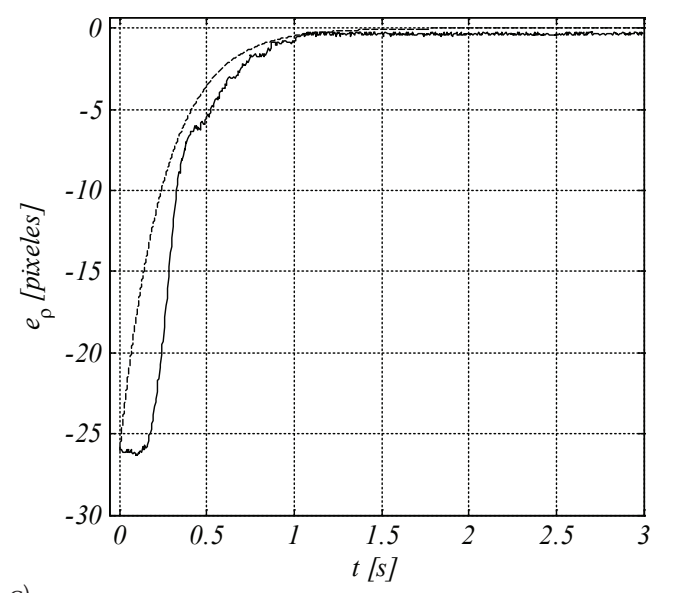

C)

Figura 3. Gráficas de la simulación (trazo discontinuo) y del experimento (trazo continuo) para el escenario 1
La figura 3 muestra las gráficas comparativas entre lo simulado y lo experimentado para el escenario 1 . La figura 3a presenta, en el plano de imagen, $y_{a l}$ mediante un círculo, $y_{b l}$ a través de un punto (ambas correspondientes al robot líder que no se mueve con una orientación de $43.31^{\circ}$, figura 2 ) y la traza del movimiento de $y_{a s}$ de manera que puede apreciarse (en el experimento) que conforme avanza el robot seguidor $y_{a s}$ se mueve de $y_{a s}(0)=\left[\begin{array}{ll}176.7 & 20.0\end{array}\right]^{T}$ [píxeles] hasta $y_{a s}(3)=\left[\begin{array}{lll}149.6 & 62.1\end{array}\right]^{T}$ [píxeles]. Esto corresponde a una $\rho(3) \approx 70.3$ [píxeles] y a una $\alpha(3) \approx 89.4^{\circ}$, valores muy cercanos a los deseados (en la simulación los errores son prácticamente nulos). Las figuras 3b, 3c y 3d ilustran la evolución contra el tiempo de las señales de control del robot seguidor, de la señal de error $e_{\rho}$ y de la señal de error $e_{\alpha^{\prime}}$ respectivamente. Como puede notarse, el desempeño es muy satisfactorio y las gráficas comparativas entre lo simulado y lo experimentado son muy similares.

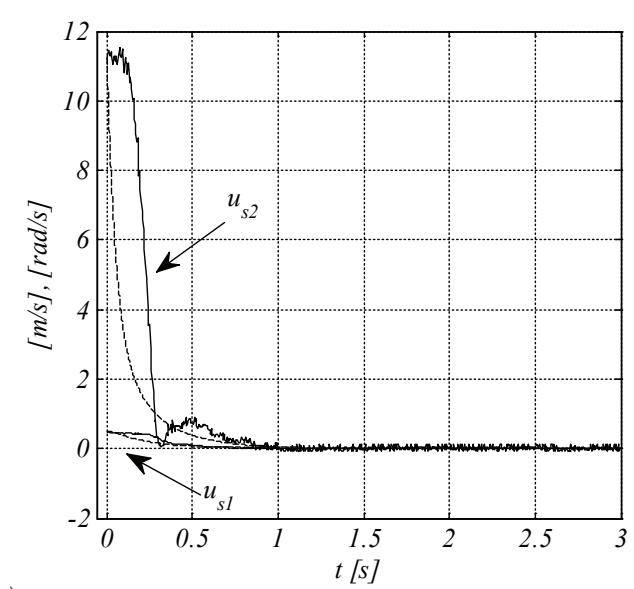

b)

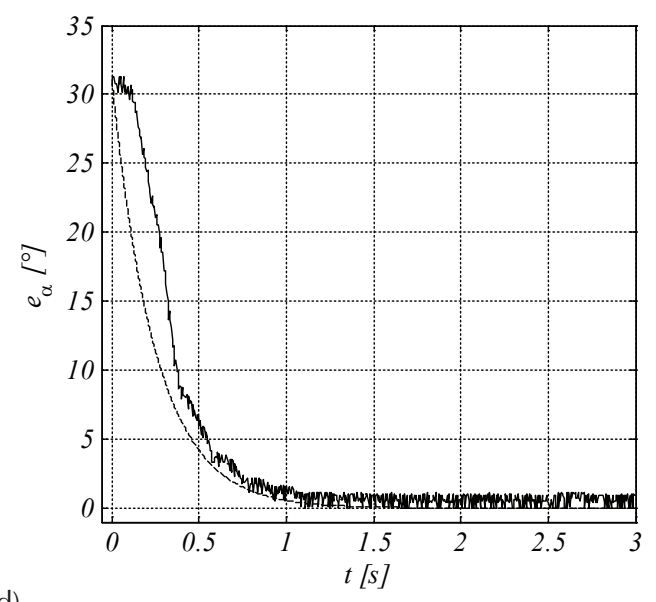

d) 


\section{Escenario 2}

En el experimento del escenario 2 se pide el siguiente vector de velocidades al robot líder: $u_{l}=[0.05 \mathrm{~m} / \mathrm{s} 0 \mathrm{rad} / \mathrm{s}]^{T}$, partiendo de las condiciones iniciales expresadas a continuación:

$x_{W I}(0)=\left[\begin{array}{lll}-0.1835 \mathrm{~m} & -0.1203 \mathrm{~m} & 34.67^{\circ}\end{array}\right] \mathrm{T}$,

$x_{W_{s}}(0)=\left[\begin{array}{lll}-0.3392 \mathrm{~m} & 0.1976 \mathrm{~m} & -20.22^{\circ}\end{array}\right] T$.

Estas condiciones iniciales corresponden a (en píxeles) $y_{a l}(0)=\left[\begin{array}{ll}105.1 & 156.6\end{array}\right]^{T}, y_{b l}(0)=\left[\begin{array}{ll}93.9 & 163.3\end{array}\right]^{T}, y_{a s}(0)=\left[\begin{array}{ll}51.1 & 56.4\end{array}\right]^{T}$, $y_{b s}(0)=[39.449 .6]^{T}, \rho(0)=112.9$ y $\alpha(0)=83.89^{\circ}$.

La figura 4a describe la traza, del experimento y de lo simulado, en el plano de imagen de la evolución tanto de $y_{a s}$ como de $y_{a l i}$ en el experimento $y_{a s}$ parte de $y_{a s}(0)=\left[\begin{array}{ll}51.1 & 56.4\end{array}\right]^{T}$ [píxeles] y termina en $y_{a s}(7)=$ [169.5 24.0 $]^{T}$ [píxeles] en tanto que $y_{a l}$ inicia en $y_{a l}(0)=$ $\left[\begin{array}{ll}105.1 & 155.6\end{array}\right]^{T}$ [píxeles] y termina en $y_{a l}(7)=\left[\begin{array}{lll}210.5 & 81.0\end{array}\right]^{T}$. Esto corresponde a una $\rho(7) \approx 70.1$ [píxeles] y a una $\alpha(7)$ $\approx 90.5^{\circ}$, los cuales son valores bastante aproximados a los deseados (nuevamente, en la simulación los errores son prácticamente nulos). Cabe destacar que el desempeño experimental es bastante satisfactorio y muy similar a lo simulado. Sin embargo, nótese que existen pequeñas diferencias entre lo simulado y lo experimentado en las trazas de $y_{a l}$ (que corresponden al robot líder); lo anterior se debe a que el robot líder se mueve en lazo abierto y no se compensan las posibles perturbaciones presentes (derrapes, deslizamientos o desperfectos en el camino no modelados). También hay diferencias, al inicio del experimento respecto a lo simulado, en la traza de $y_{a s}$ las cuales también se deben a los posibles derrapes, deslizamientos y desperfectos en el camino, pero que ahora sí están compensadas por el controlador. Lo anterior se puede ver en la figura $4 \mathrm{~b}$ que gráfica la evolución respecto al tiempo de las seña-
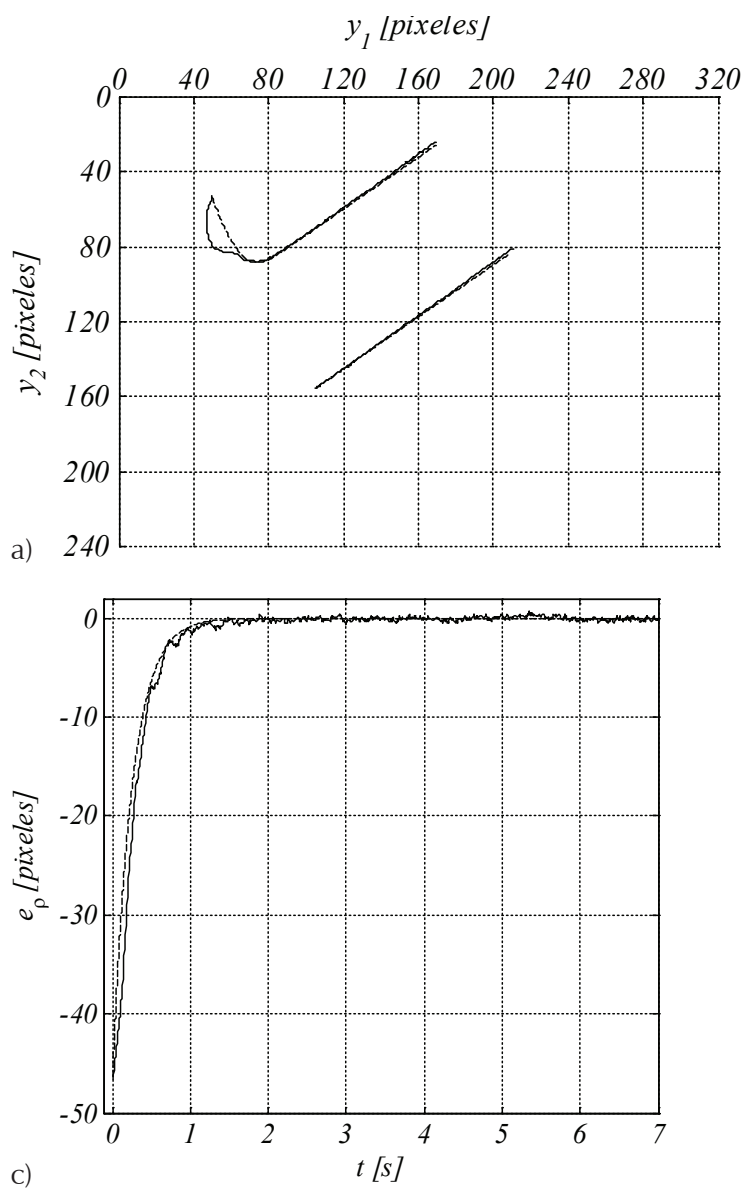

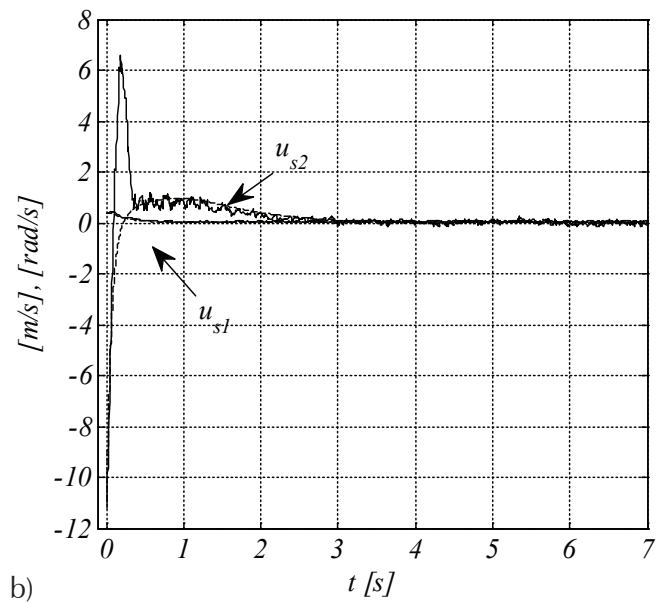

b)

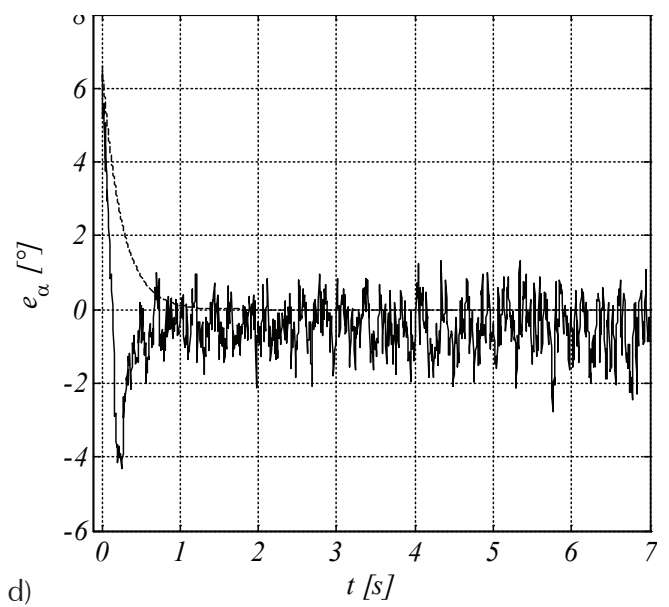

Figura 4. Gráficas de la simulación (trazo discontinuo) y del experimento (trazo continuo) para el escenario 2 
les de control y en donde se observa un sobreimpulso en la señal de control $u_{\mathrm{s} 2}$ para solventar dichas situaciones. Las figuras 4c y 4d ilustran la evolución contra el tiempo de los errores de formación. En forma general se puede decir que el desempeño del sistema robótico con el controlador visual propuesto es muy satisfactorio, resaltándose que las gráficas en comparación (experimentos con simulaciones) son muy similares.

\section{Conclusiones}

Se describió la propuesta de un control visual para la formación de robots móviles tipo uniciclo bajo el esquema líder-seguidor que no depende de los parámetros (intrínsecos o extrínsecos) del sistema de visión. Para este efecto, se consideró una sola cámara fija observando el espacio de trabajo de los robots que, en términos de la información procesada, se puede compartir tanto por el robot líder como por el robot seguidor. Lo anterior permite que la implementación de esta propuesta pueda llevarse a cabo por estrategias de control centralizadas o descentralizadas. Ahora bien, el objetivo de control de formación se establece directamente en coordenadas de imagen. Cabe hacer notar que el controlador (además de mediciones en el espacio de imagen) solamente requiere el conocimiento de las velocidades del robot líder y de un parámetro establecido por el propio usuario; ambos requerimientos pudieran estimarse (ver por ejemplo, Dani et al., (2009)) o solventarse por alguna ley de adaptación (lo que se considera como un trabajo futuro, para efectos de este documento). Finalmente, también como parte importante de este trabajo, se detallan experimentos satisfactorios utilizando un sistema de visión de tiempo real y alta velocidad para validar la teoría propuesta con un periodo de muestreo estricto de 0.005 [s].

\section{Agradecimientos}

Se agradece el apoyo de CONACYT México (Proyecto 166654), PROMEP, DGEST y al Instituto Tecnológico de Ensenada.

\section{Referencias}

Antonelli G., Arrichiello F., Chiaverini S. Experiments of Formation Control with Collision Avoidance Using the Null-Space-based Behavioral Control, Proceedings of IEEE Mediterranean Conference on Control and Automation, Ancona, Italy, 2006, pp. 1-6.

Balch T. y Arkin R.C. Behavior-Based Formation Control for Multirobot Teams. IEEE Transactions on Robotics and Automation, volumen 14 (número 6), 1998: 926-939.
Belta C. y Kumar V. Trajectory Design for Formations of Robots by Kinetic Energy Shaping, Proceedings of IEEE International Conference on Robotics and Automation, Washington, USA, 2002, pp. 2593-2598.

Benhimane S., Malis E., Rives P., Azinheira J.R. Vision-Based Control for Car Platooning Using Homography Decomposition. Proceedings of the 2005 IEEE International Conference on Robotics and Automation, Barcelona, Spain, 2005. pp. 2161-2166.

Brockett R.W. Asymptotic Stability and Feedback Stabilization, en: Brockett R.W., Millman R.S., Sussmann H.J., Eds. Differential Geometric Control Theory, Birkhäuser, Boston, MA, 1983, pp. 181-191.

Bugarin E. y Kelly R. Sobre el control servo-visual para la navegación de un robot uniciclo independiente de los parámetros de la cámara. The Anáhuac Journal, volumen 8 (número 2), 2008: 57-68.

Canudas de Wit, C., Siciliano B., Bastin G. Theory of Robot Control, Springer-Verlag, London, U.K., 1996.

Consolini L., Morbidi F., Prattichizzo D., Tosques M. On the Control of a Leader-Follower Formation of Nonholonomic Mobile Robots, Proceedings of the 45th IEEE Conference on Decision \& Control, San Diego, CA, USA, 2006, pp. 5992-5997.

Conticelli F., Allotta B., Khosla P.K. Image-Based Visual Servoing of Nonholonomic Mobile Robots, Proceedings of the 38th Conference on Decision and Control, Phoenix, Arizona, USA, diciembre de 1999, pp. 3496-3501.

Dani A.P., Gans N., Dixon W.E. Position-Based Visual Servo Control of Leader-Follower Formation Using Image-Based Relative Pose and Relative Velocity Estimation, American Control Conference. St. Louis, MO, USA, junio de 2009, pp. 5271-5276.

Das A.K., Fierro R., Kumar V., Ostrowsky J.P., Spletzer J., Taylor C. A Vision-Based Formation Control Framework. IEEE Transaction on Robotics and Automation, volumen 18 (número 5), 2002: 813-825.

Desai J., Ostrowski J., Kumar V. Modelling and Control of Formations of Nonholonomic Mobile Robots. IEEE Transaction on Robotics and Automation, volumen 17, 2001: 905-908.

Dixon W., Dawson D., Zergeroglu E., Behal A. Nonlinear Control of Wheeled Mobile Robots, Springer, London, U.K., 2001.

Fang Y., Dixon W.E., Dawson D.M., Chawda P. Homography-Based Visual Servo Regulation of Mobile Robots. IEEE Transactions on Systems, Man, and Cybernetics, Part B: Cybernetics, volumen 35 (número 5), 2005: 1041-1050.

Hashimoto K. y Toshiro N. Visual Servoing of Nonholonomic Cart, Proceedings of the 1997 IEEE International Conference on Robotics and Automation, Albuquerque, NM, abril de1997, pp. 1719-1724.

Hill J. y Park W.T. Real Time Control of a Robot with a Mobile Camera, 9th ISIR, Washington, D.C., marzo de 1979, pp. 233-246.

Hutchinson S., Hager G., Corke P. A Tutorial on Visual Servoing. IEEE Transactions on Robotics and Automation, volumen 12 (número 5), 1996: 651-670. 
Kelly R. y Reyes F. On Vision Systems Identification with Application to Fixed Camera Robotics Systems. International Journal of Imaging Systems and Technology, volumen 11 (número 3), 2000: 170-180.

Khalil H. Nonlinear systems, 3ed., Prentice Hall, 2001.

Lawton J.R., Beard R.W., Young B.J. A Decentralized Approach to Formation Maneuvers. IEEE Transactions on Robotic and Automation, volumen 19 (número 6), 2003.

López-Nicolás G., Sagüés C., Guerrero J.J., Kragic D., Jensfelt P. Nonholonomic Epipolar Visual Servoing, Proceedings of the 2006 IEEE International Conference on Robotics and Automation, Orlando, Florida, mayo de 2006, pp. 2378-2384.

Mehta S.S., Hu G., Gans N.R., Dixon W.E. Adaptive Vision-Based Collaborative Tracking Control of an UGV Via a Moving Airborne Camera: A Daisy Chaining Approach, Proceedings of the 45th Conference on Decision and Control, San Diego, CA, USA, diciembre de 2006, pp. 3867-3872.

Mariottini G.L., Prattichizzo D., Oriolo G. Epipole-Based Visual Servoing for Nonholonomic Mobile Robots, Proceedings of the 2004 IEEE International Conference on Robotics and Automation, New Orleans, LA., mayo de 2004, pp. 497-503.

Min H.J., Drenner A., Papanikolopoulos N. Vision-Based LeaderFollower Formations with Limited Information, IEEE International Conference on Robotics and Automation, Kobe, Japan, mayo de 2009, pp. 351-356.

Monteiro S., Vaz M., Bicho E. Attractor Dynamics Generates Robot Formations: from Ttheory to Implementation, Proceedings of IEEE International Conference on Robotics and Automation, New Orleans, USA, 2004, pp. 2582-2587.

Renaud P., Cervera E., Martinet P. Towards a Reliable Vision-Based Mobile Robot Formation Control, Proceedings of the 2004 IEEE/RSJ, International Conference on Intelligent Robots and Systems, Sendai, Japón, octubre de 2004, pp. 3176-3181.
Roberti F., Toibero J.M., Vassallo R.F., Carelli R. Control estable de formación basado en visión omnidireccional para robots móviles no holonómicos. Revista Iberoamericana de Automática e Informatica Industrial, volumen 8 (número 1), 2011: 29-37.

Soria C.M., Carelli R., Kelly R., Ibarra J.M. Coordinated Control of Mobile Robots Based on Artificial Vision. International Journal of Computers, Communications, and Control, volumen 1 (número 2), 2006: 85-94.

Shao J., Xie G., Yu J., Wang L. Leader-Following Formation Control of Multiple Mobile Robots, Proceedings of IEEE International Symposium on Intelligent Control, Limassol, Cyprus, 2005, pp. 808-813.

Spong M.W., Hutchinson S., Vidyasagar M. Robot Modeling and Control, Wiley, USA, 2006.

Tan K. y Lewis M. Virtual Structures for High-Precision Cooperative Mobile Robotic Control. Autonomous Robots, volumen 4, 1997: 387-403.

\section{Este artículo se cita: \\ Citación estilo Chicago}

Bugarin-Carlos, Eusebio, Ana Yaveni Aguilar-Bustos. Control visual para la formación de robots móviles tipo uniciclo bajo el esquema líder-seguidor. Ingeniería Investigación y Tecnología, XV, 04 (2014): 593-602.

\section{Citación estilo ISO 690}

Bugarin-Carlos E., Aguilar-Bustos A.Y. Control visual para la formación de robots móviles tipo uniciclo bajo el esquema líder-seguidor. Ingeniería Investigación y Tecnología, volumen XV (número 4), octubre-diciembre 2014: 593-602.

\section{Semblanza de los autores}

Eusebio Bugarin-Carlos. Recibió el título de ingeniería en el Instituto Tecnológico de Tijuana (ITT) en 1995. Luego, en 1998, obtuvo el título de maestría en ciencias en ingeniería eléctrica por el Instituto Tecnológico de La Laguna (ITL) y, posteriormente, en 2009 el grado de doctor en ciencias en electrónica y telecomunicaciones por el Centro de Investigación Científica y de Educación Superior de Ensenada (CICESE). Actualmente es profesor-investigador en el Instituto Tecnológico de Ensenada y sus áreas de interés incluyen control de robots, formación de robots y visión artificial.

Ana Yaveni Aguilar-Bustos. En 1995 obtuvo el título de ingeniería por el Instituto Tecnológico de Tijuana (ITT). Después, recibió tanto el título de maestría en ciencias en electrónica y telecomunicaciones (en 1998) como el grado de doctor en ciencias en electrónica y telecomunicaciones (en 2006) en el Centro de Investigación Científica y de Educación Superior de Ensenada (CICESE). Actualmente es profesor-investigador en el Instituto Tecnológico de Ensenada y sus principales áreas de interés son sistemas no-lineales, sistemas complejos y caos. 